\title{
Reductions in Drag and Fluctuating Forces for a Circular Cylinder by Attaching Cylindrical Rings"
}

\author{
Hajime NAKAMURA $^{* *}$ and Tamotsu IGARASHI ${ }^{* *}$ \\ **National Defense Academy, \\ 1-10-20 Hashirimizu, Yokosuka-shi, Kanagawa, Japan \\ E-mail: nhajime@nda.ac.jp
}

\begin{abstract}
Omnidirectional reductions in drag and fluctuating forces were achieved for a circular cylinder subjected to cross-flow by attaching cylindrical rings along its span at an interval of several diameters. The Reynolds number based on the cylinder diameter, $d$, ranged from $R e_{d}=3000$ to 38000 for the experiments. The aspect ratio of the cylinder, $L / d$, was approximately 20 . The addition of the rings reduced the drag force for $R e_{d} \geq 20000$, even though the projected area increased. This was attributed to the formation of separation bubbles on both sides of the ring, which led to pressure recovery on the rear of the cylinder. The optimum ring configuration for drag reduction was found to be $D / d=1.3, W / d=1.0$, and $P / d=6$ at $R e_{d} \approx 30000$, where $D$ is the ring diameter, $W$ is the spanwise width of the ring, and $P$ is the spanwise pitch of the ring. This configuration reduced the drag force by $15 \%$. In addition, the fluctuating lift, which was estimated from the fluctuating surface pressures, was weakened for all Reynolds numbers due to the suppression of vortex shedding.
\end{abstract}

Key words: Passive Flow Control, Drag Force, Fluctuating Force, Circular Cylinder, Vortex Shedding, Three-Dimensional Flow, Streamwise Vortices

\section{Introduction}

A two-dimensional bluff bodies subjected to cross-flow generates a three-dimensional wake by forming streamwise vortices in the spanwise vortex street (Ref. (1) and (2) among others). In connection to this, it is suggested that the vortex shedding can be suppressed by introducing streamwise vortices through three-dimensional surface modifications. Bearman and Owen ${ }^{(3)}$ and Owen et al. ${ }^{(4)}$ investigated drag reduction and vortex shedding suppression of a rectangular cylinder and a circular cylinder by introducing wavy separation lines. That is, a sinusoidal wavy front face was added to a cylinder with a rectangular cross-section and a sinusoidal wavy axis was added to a cylinder with a circular cross-section. As a result, the drag coefficient was reduced up to $30-47 \%$ compared to the unmodified straight cylinders, even though the amplitude of the waviness was small; the ratio of the peak-to-peak wave height divided by the wavelength was approximately 0.1. Darekar and Sherwin ${ }^{(5)}$ investigated the flow past a square cross-section cylinder with a sinusoidal wavy axis for Reynolds numbers up to 150 using numerical simulations. The results indicated that the minimum wave amplitude for vortex shedding suppression was achieved when the wavelength of the waviness was close to the mode-A wavelength ${ }^{(1)}$, which is equivalent to five to six times the length of a side of the square cross-section.

Although these modifications dramatically reduce drag and suppress vortex shedding, they are only effective for a given flow direction relative to the cylinder. The next step is to develop geometries that are effective for all flow directions. Owen et al. ${ }^{(4)}$ attached 
hemi-spherical bumps in a spiral pattern on the surface of a circular cylinder. This geometry

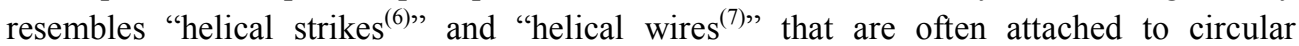
cylinders to suppress flow-induced vibration. Although these features were not initially designed for drag reduction, this geometry proposed by Owen et al. reduced the drag coefficient by $25 \%$ and suppressed the vortex shedding. Ahmed et al. ${ }^{(8)(9)}$ studied the flow around a wavy circular cylinder. The diameter of the circular cross-section changed in a sinusoidal manner along the span of the cylinder. For this configuration, streamwise vortices formed near the geometric nodes of the cylinder, but the reduction of the drag coefficient was small, only $9 \%$ at maximum. Lam et al. ${ }^{(10)(11)}$ investigated the wavy circular cylinder for a larger aspect ratio. For this experiment, the axial length divided by the mean diameter was approximately 27 , and the drag coefficient was reduced by up to $20 \%$.

In the present study, cylindrical rings were attached to a circular cylinder, as shown in Fig. 1. The advantages of this geometry are its axial symmetry and its simplicity for manufacturing. The proposed configuration reduced the drag coefficient up to $18 \%$. Flow visualizations and flow measurements were conducted in order to investigate the reasons behind the drag reduction.

\section{Nomenclature}

$A$ : projected area to the flow $=d L+n(D-d) W \quad \mathrm{~m}^{2}$

$C_{D}$ : drag coefficient $=F_{D} /\left(0.5 \rho u_{0}^{2} A\right)$

$C_{L}$ : lift coefficient $=F_{L} /\left(0.5 \rho u_{0}^{2} A\right)$

$C p$ : pressure coefficient $=\left(p-p_{0}\right) /\left(0.5 \rho u_{0}{ }^{2}\right)$

$D$ : diameter of ring $\mathrm{m}$ or $\mathrm{mm}$

$d$ : diameter of circular cylinder $\mathrm{m}$ or $\mathrm{mm}$

$F_{D}, F_{L}$ : drag and lift forces $\mathrm{N}$

$f, f_{s}$ : frequency, vortex shedding frequency $\mathrm{Hz}$

$L$ : axial length of cylinder $\mathrm{m}$ or $\mathrm{mm}$

$n$ : number of rings

$P:$ spanwise pitch of rings $\mathrm{m}$ or $\mathrm{mm}$

$p:$ static pressure on a wall $\mathrm{Pa}$

$p_{0}$ : static pressure at freestream $\mathrm{Pa}$

$R e_{d}$ : Reynolds number $=u_{0} d / \mathrm{v}$

$S t_{d}:$ Strouhal number $=f_{s} d / u_{0}$

$t:$ time sec

$u, u_{0}$ : velocity, freestream velocity $\mathrm{m} / \mathrm{s}$

$W$ : spanwise width of ring $\mathrm{m}$ or $\mathrm{mm}$

$x, y, z:$ streamwise, cross-stream, and spanwise coordinates (see Fig. 1)

$\phi:$ angle from the forward stagnation point ${ }^{\circ}$

$v, \rho$ : kinematic viscosity $\left(\mathrm{m}^{2} / \mathrm{s}\right)$ and density of fluid $\left(\mathrm{kg} / \mathrm{m}^{3}\right)$ subscripts

$2 D:$ two-dimensional cylinder

ave, $r m s$ : time-averaged value, rms value

\section{Experimental Apparatus}

Figure 1 shows the schematic diagram and symbols for the test model. The wind tunnel has a $400 \mathrm{~mm}$ high and $150 \mathrm{~mm}$ wide working section. The test cylinder was placed at the wind tunnel exit in order to reduce the blockage effect. The cylinder was fabricated from acrylic-resin pipe and had a diameter $d$ of either 15 or $20 \mathrm{~mm}$ and a length $L$ of $360 \mathrm{~mm}$. Square end plates, $150 \mathrm{~mm}$ in length, were attached to both ends of the cylinder. The 
freestream velocity $u_{0}$ ranged from 3 to $30 \mathrm{~m} / \mathrm{s}$, resulting in Reynolds numbers based on $d$ ranging between 3000 and 38000. The turbulent intensity of the freestream was approximately $0.5 \%$. Acrylic resin cylindrical rings were attached to the cylinder. In order to fully investigate the effects of the ring configurations, the diameter $D$, spanwise width $W$, and spanwise pitch $P$ were systematically varied in the ranges of $1.2 \leq D / d \leq 2.0,0.33 \leq W / d \leq$ 4.0 , and $2 \leq P / d \leq 9$, respectively.

The drag force was measured

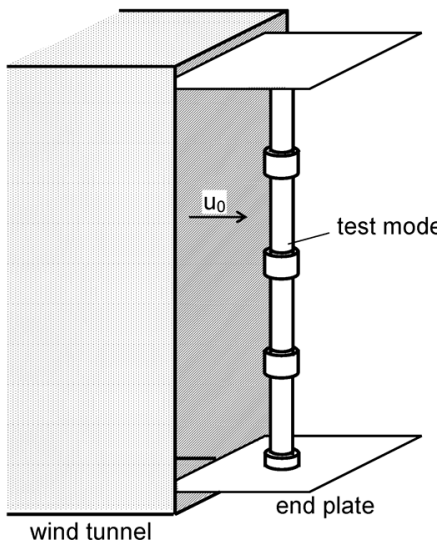

(a) schematic diagram

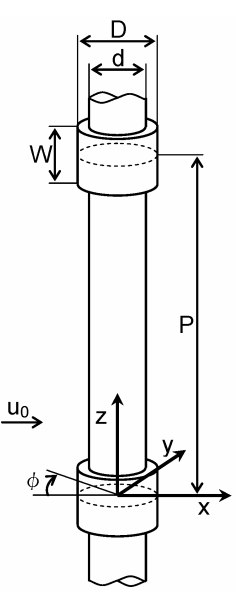

(b) symbols

Fig. 1 Ring-attached cylinder

using a three-component balance (LMC-3504, Nissho) that was installed below the test cylinder. In this configuration, the lower endplate had a hole larger than the test cylinder diameter, so the balance was used to support the lower end of the cylinder. The upper end of the cylinder and the upper endplate were separated by approximately $0.5 \mathrm{~mm}$. The portion of the cylinder that protruded below the lower endplate was enclosed within a pipe to prevent the fluid from acting on it.

The pressure and velocity measurements as well as the flow visualizations were performed with both ends of the cylinders affixed to the endplates. Two pressure transducers (PD104K-10K, Toyoda) buried inside the test cylinder were used to measure the surface pressures on the cylinder. Two $1.5 \mathrm{~mm}$ diameter pressure holes, each connected to a transducer, were constructed directly across from each other at the mid-span of the cylinder. These holes were used to simultaneously measure the fluctuating pressure on both sides of the cylinder $\left(\phi= \pm 90^{\circ}\right)$. The resonance frequency of the pressure holes was calculated to be approximately $2 \mathrm{kHz}$, which indicated that frequencies below $400 \mathrm{~Hz}$ could be measured without accounting for the resonance effect. The circumferential and spanwise positions of the pressure holes were modified by rotating the cylinder around its axis and by sliding the rings along its span, respectively.

\section{Drag Reduction}

\subsection{Effect of the ring configuration}

Figures 2 (a) - (c) show the effects of diameter $D / d$, spanwise width $W / d$, and spanwise pitch $P / d$, on the drag force $F_{D} / F_{D 2 D}$ at $R_{d} \approx 30000 . F_{D}$ is the drag force acting on the ring-attached cylinder and $F_{D 2 D}$ is the drag force acting on the 2D cylinder without rings. A three-component balance was employed to obtain these measurements. The diameter of the base cylinder was either $d=15 \mathrm{~mm}(L / d=24)$ or $d=20 \mathrm{~mm}(L / d=18)$. The projected area of the ring-attached cylinder to the flow, $A$, is defined as

$$
A=d L+n(D-d) W
$$

where $n$ is the number of rings. Although the projected area increases when more rings are attached, the drag force is reduced compared to that of a $2 \mathrm{D}$ cylinder without rings, as shown in Fig. 2 . The minimum drag force occurs at $D / d \approx 1.3, W / d \approx 1.0$, and $P / d \approx 6$ for both aspect ratios. The drag reduction is less significant for the smaller aspect ratio $L / d=$ 18. This is probably due to the large three-dimensionality in the wake for the smaller aspect 


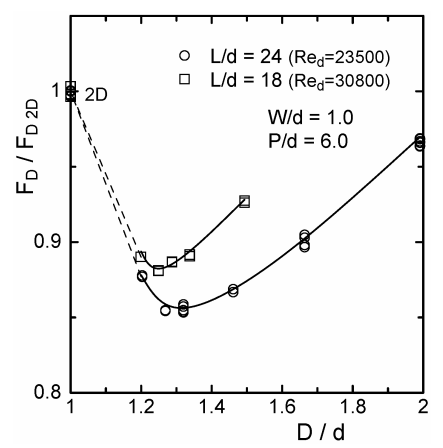

(a) diameter $D / d$

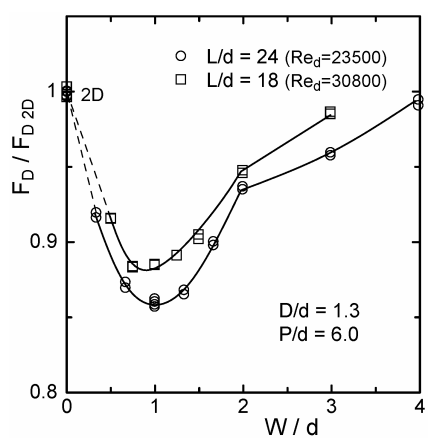

(b) spanwise width $W / d$

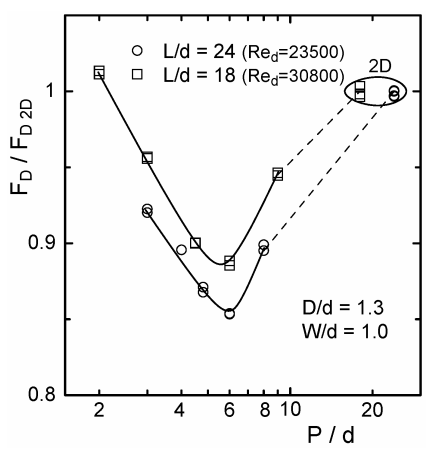

(c) spanwise pitch $P / d$

Fig. 2 Effect of ring configuration on the drag force at $R e_{d} \approx 30000$

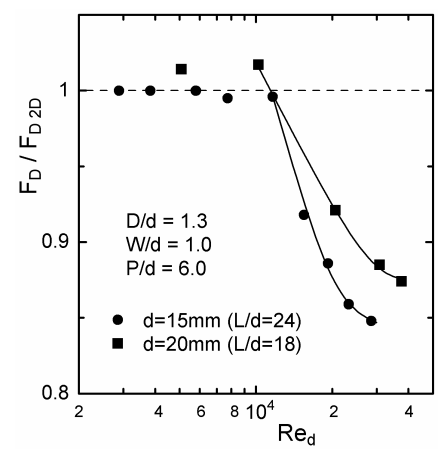

(a) drag force $F_{D} / F_{D 2 D}$

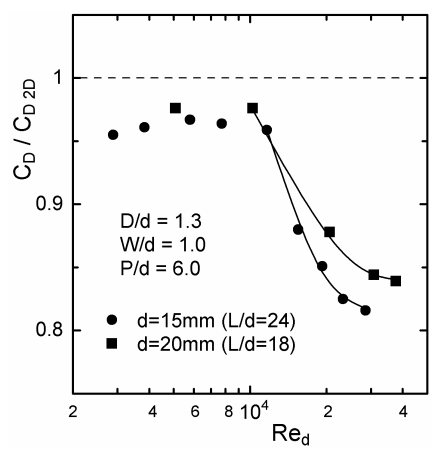

(b) drag coefficient $C_{D} / C_{D 2 D}$

Fig. 3 Effect of Reynolds number on the drag for the optimum ring configuration $(D / d=1.3, W / d=1.0, P / d=6)$

ratio cylinder (Ref. (12) among others), which results in the weakening of the three-dimensional effects by attaching rings.

\subsection{Effect of the Reynolds number}

Figures 3 (a) and (b) show the effects of the Reynolds number $R e_{d}$ on the drag force, $F_{D} / F_{D 2 D}$, and the drag coefficient defined by the projected area, $C_{D} / C_{D 2 D}$, respectively, for the optimum ring configuration $(D / d=1.3, W / d=1.0$, and $P / d=6)$. Although the drag reduction is relatively insignificant for lower Reynolds numbers $\left(R e_{d} \leq 10000\right)$, it suddenly drops in the $10000<R e_{d} \leq 20000$ range and then continues to gradually decrease for $R e_{d}>$ 20000. The current experimental conditions realized a $15 \%$ reduction in the drag force $F_{D}$ and an $18 \%$ reduction in the drag coefficient $C_{D}$ for the larger aspect ratio $(L / d=24)$ cylinder and a high, but still sub-critical, Reynolds number $\left(R e_{d}=29000\right)$.

\subsection{Changes in the flow by attaching rings}

In order to investigate the reasons behind the drag reduction for $R e_{d}>10000$, flow visualizations were performed at $R e_{d}=10000$ and 20000. Figure 4 shows spanwise flow visualizations using the smoke-wire method. Placing a thin wire at an asymmetrical position (at $x / d=-1.5$ and $y / d=0.25$ ) allows for the visualization of only the opposite side of the shear layer. As shown in Fig. 4 (a), the vortex shedding behind the 2D cylinder is generally two-dimensional and the spanwise undulation is caused by the streamwise vortices (Ref. (2) and (13) among others). These features are evident at both $R e_{d}=10000$ and 20000. On the other hand, the flow behind the ring-attached cylinder changes remarkably between $R e_{d}=$ 10000 and 20000, as shown in Fig. 4 (b). Although the vortex shedding at $R e_{d}=10000$ is basically the same as that for the $2 \mathrm{D}$ cylinder, it is evident that the spanwise vortex tube bifurcates behind the ends of the upper ring (Fig. 4(b), $R e_{d}=10000$ ). This can be attributed 


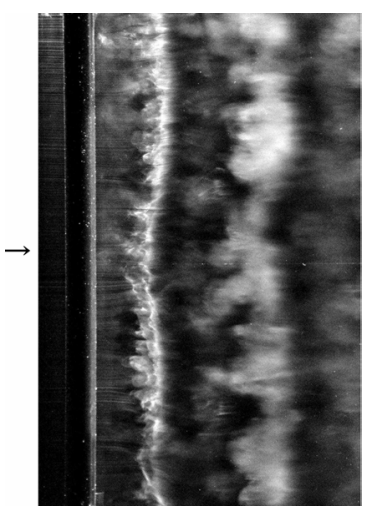

$R e_{d}=10000$

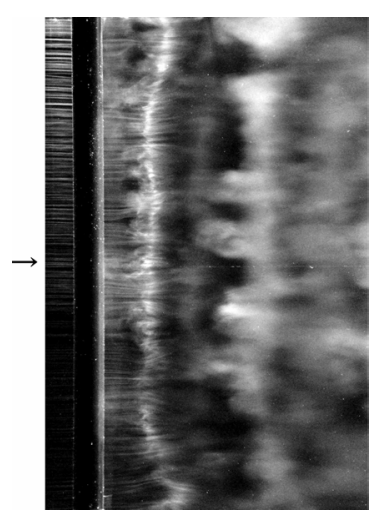

$R e_{d}=20000$

(a) $2 \mathrm{D}$ cylinder

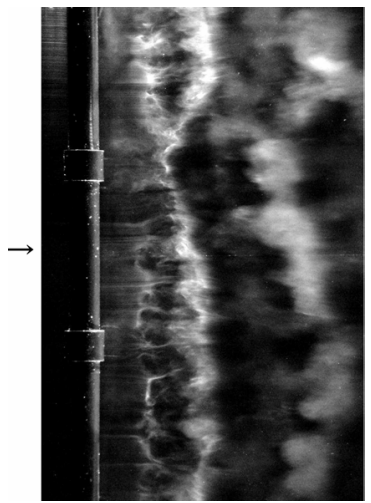

$R e_{d}=10000$

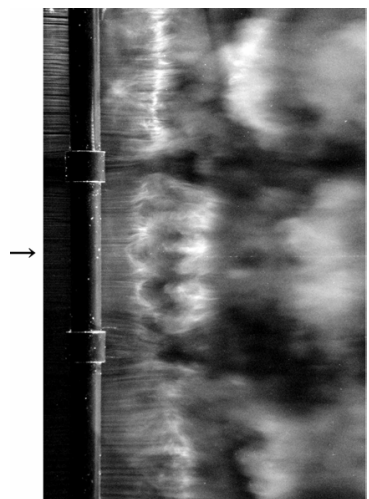

$R e_{d}=20000$

(b) ring-attached cylinder $(D / d=1.3, W / d=1.0, P / d=6)$

Fig. 4 Spanwise flow visualizations using the smoke-wire method behind the cylinder $(L / d=18)$

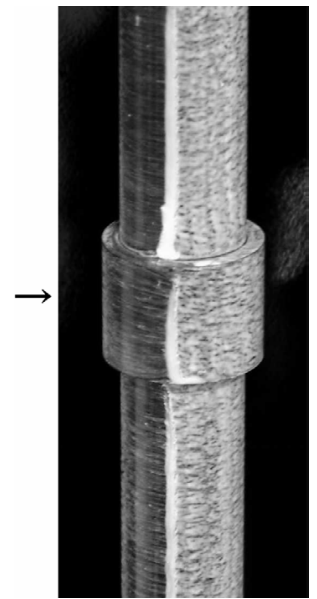

(a) $R e_{d}=10000$

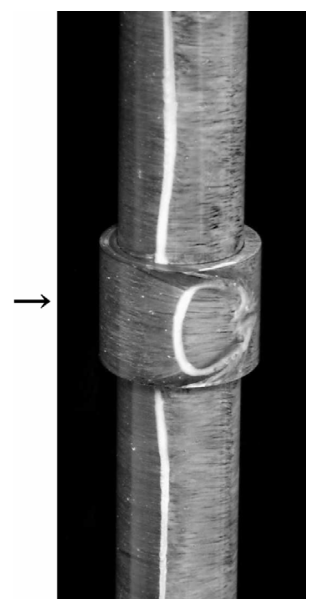

(b) $R e_{d}=20000$

Fig. 5 Surface oil-film patterns around the ring-attached cylinder

$$
(L / d=18, D / d=1.3, W / d=1.0, P / d=6)
$$

to the different shedding frequencies for the base cylinder and the ring. In contrast, at $R e_{d}=$ 20000, the spanwise vortex tube is completely divided at the ring locations, exhibiting a highly three-dimensional flow behind the cylinder.

Figure 5 shows the surface oil-film pattern around the ring. The separation line at $R e_{d}=$ 10000 is nearly two-dimensional for both the base cylinder and the ring, with a slight discontinuity in the separation angle at both edges of the ring. At $R e_{d}=20000$, however, the 


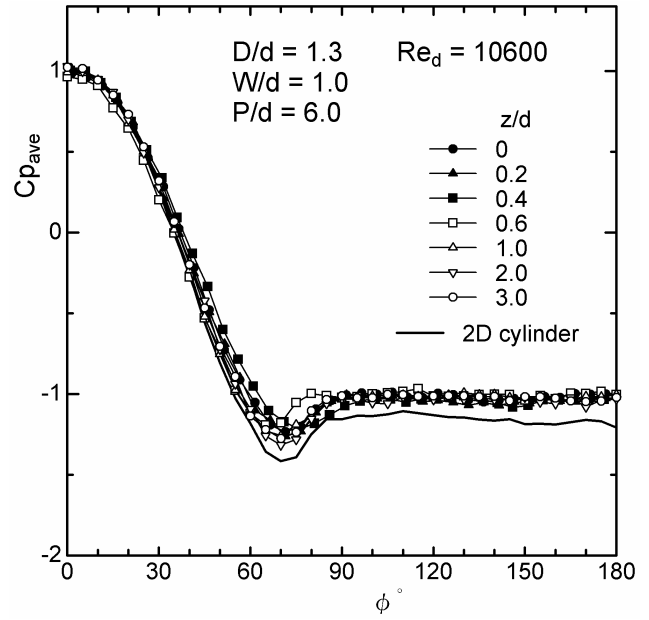

(a) $R e_{d}=10600$

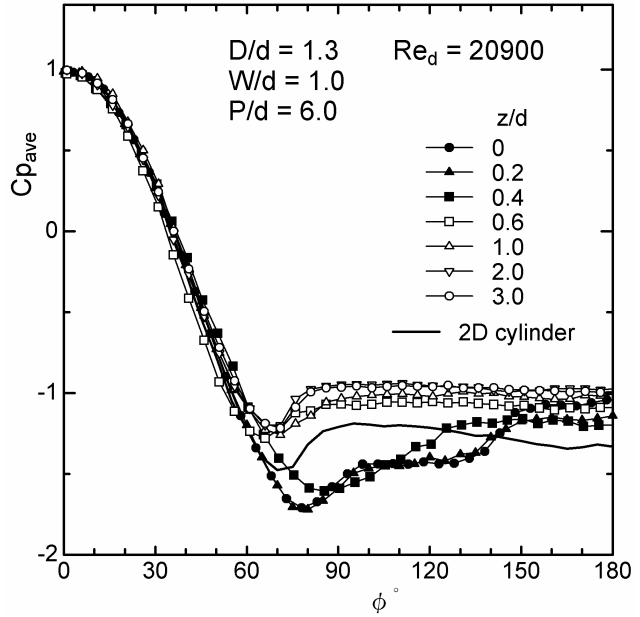

(b) $R e_{d}=20900$

Fig. 6 Time-averaged pressure distributions around the ring-attached cylinder $(L / d=18, D / d=1.3, W / d=1.0, P / d=6)$

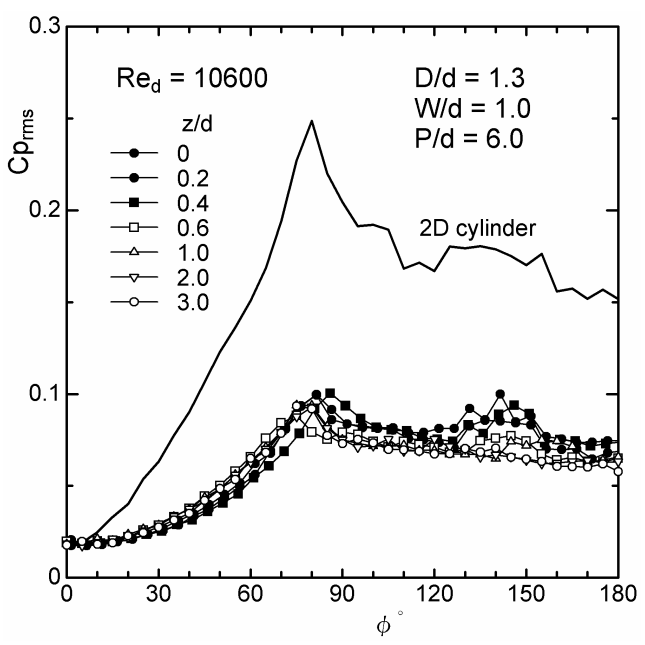

(a) $R e_{d}=10600$

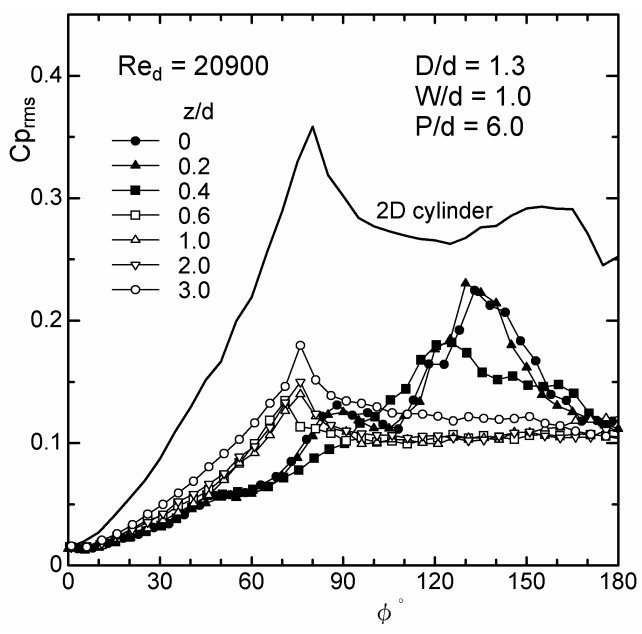

(b) $R e_{d}=20900$

Fig. 7 Fluctuating pressure distributions around the ring-attached cylinder $(L / d=18, D / d=1.3, W / d=1.0, P / d=6)$

separation line on the ring is markedly distorted into a highly three-dimensional feature, exhibiting a backward movement of the separation line on the ring. Also, the separated flow from the side of the ring reattaches at the rear of the ring $\left(\phi \approx 135^{\circ}\right)$, indicating the formation of a separation bubble on the side of the ring.

Figures 6 and 7 depict the pressure distributions around the ring-attached cylinder. Figure 6 shows the time-averaged pressure coefficient $C p_{\text {ave }}$ and Figure 7 shows the rms value of the fluctuating pressure coefficient $C p_{r m s}$ at both $R e_{d} \approx 10000$ and $R e_{d} \approx 20000$. The solid symbols in these Figures are on the ring and the open symbols are on the base cylinder. At $R e_{d} \approx 10000$, both $C p_{\text {ave }}$ and $C p_{r m s}$ change only slightly along the axis, exhibiting quasi-two-dimensional distributions. The flow separation point, which occurs at the maximum value in the $C p_{\text {rms }}$ distribution (Fig. 7 (a), $\phi \approx 80^{\circ}$ ), shifts backward near the ring edges $(z / d=0.4)$ and shifts forward on the base cylinder adjacent to the ring edges $(z / d$ $=0.6$ ), which are consistent with the oil-film pattern in Fig. 5(a). In contrast, the distributions of both $C p_{a v e}$ and $C p_{r m s}$ at $R e_{d} \approx 20000$ change dramatically between the ring $(z / d=0-0.5)$ and the base cylinder $(z / d=0.5-3)$. The $C p_{r m s}$ distribution on the ring has two maximums, at $\phi \approx 90^{\circ}$ and $\phi \approx 135^{\circ}$, which correspond to the flow separation and 


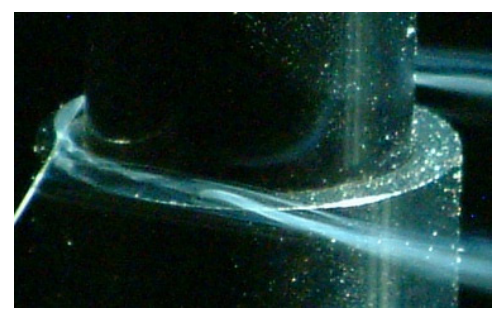

(a) $R e_{d}=10000$

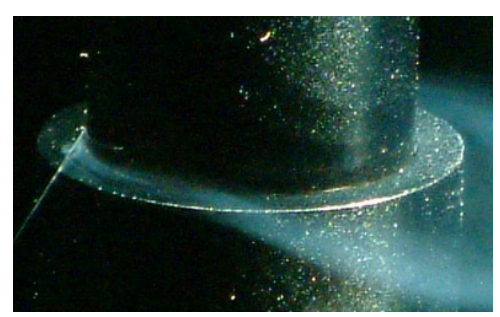

(b) $R e_{d}=20000$

Fig. 8 Junction vortex formed at the ring edge

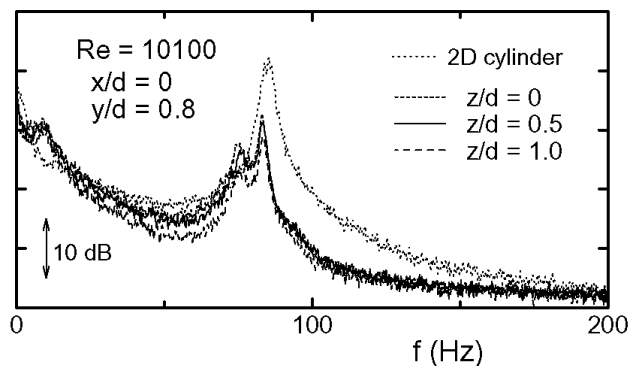

(a) $R e_{d}=10100$

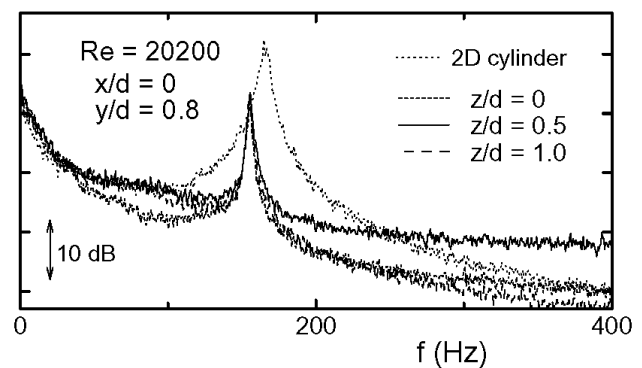

(b) $R e_{d}=20200$

Fig. 9 Power spectrum of the velocity fluctuation $(x=0, y / d=0.8)$

reattachment points, respectively. This indicates the formation of a separation bubble on the side of the ring in the region of $90^{\circ} \leq \phi \leq 135^{\circ}$, which is evident in the surface oil-film pattern in Fig. 5(b). A decrease in the $C p_{\text {ave }}$ value on the side of the ring (Fig. 6(b), $70^{\circ} \leq \phi$ $\left.\leq 140^{\circ}\right)$ is due to the backward movement of the flow separation point on the ring $\left(\phi \approx 90^{\circ}\right)$ compared to the 2D cylinder $\left(\phi \approx 80^{\circ}\right)$.

It should be noted that the flow on the ring itself for $R e_{d} \geq 20000$ is similar to that around a circular cylinder in the critical regime $\left(3 \times 10^{5}<R e_{d}<1.5 \times 10^{6}\right)$. The formation of the separation bubbles causes the wake to narrow, as shown later in Fig. 11(b), and the pressure to recover on the rear of the ring. This, in turn, results in the drag reduction. Based on these results, it is reasonable to assume that the turbulent transition in the boundary layers occurs on the ring at the lower Reynolds numbers, between $R e_{d}=10000$ and 20000 . The pressure recovery behind the ring, however, is smaller than that for the 2D cylinder in the critical regime. This is due to the formation of spanwise flow which equalizes the base pressure along span, as shown in Fig. 6 (b).

Figure 8 shows flow visualizations around the ring edge using the smoke-wire method. As shown in Fig. 8 (a), a junction vortex, which is similar to the horseshoe vortex formed around a three-dimensional protuberance, is formed and it turns streamwise vortices at both sides. At $R e_{d}=10000$, streamlines of the vortex is clearly identified around the ring, though it becomes unclear at $R e_{d}=20000$. This suggests that the turbulent transition occurs around the ring edge between $R e_{d}=10000$ and 20000. Figure 9 shows power spectrum of the velocity fluctuation at the side of the ring $(x=0, y / d=0.8)$ measured using an I-type hot wire probe. At $R e_{d} \approx 20000$, there is a noticeable change in the power spectrum at the ring edge $(z / d=0.5)$, at which the higher frequency component increases markedly. This also suggests the occurrence of the turbulent transition around the ring edge between $R e_{d}=$ 10000 and 20000, which may cause the turbulent transition in the boundary layer on the side of the ring.

\subsection{Changes in the vortex shedding characteristics}

Figure 9 also indicates that the vortex shedding characteristics change between $R e_{d}=$ 10000 and 20000. That is, the power spectrum at $R e_{d} \approx 10000$ has two distinct peaks at the vortex shedding frequency, while it has a single peak at $R e_{d} \approx 20000$. Figure 10 shows the 


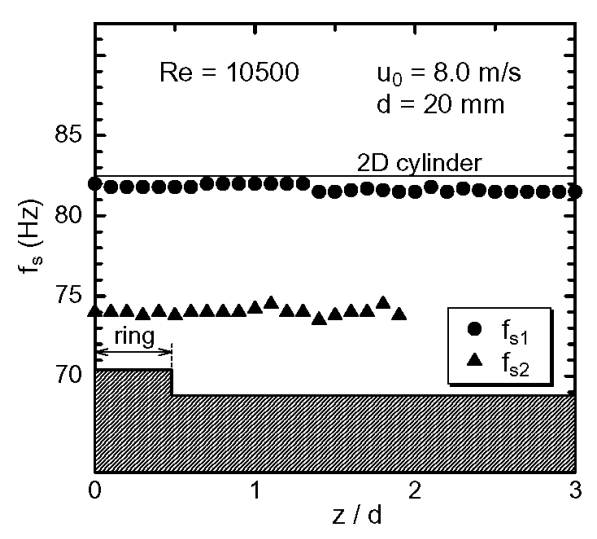

(a) $R e_{d}=10500$

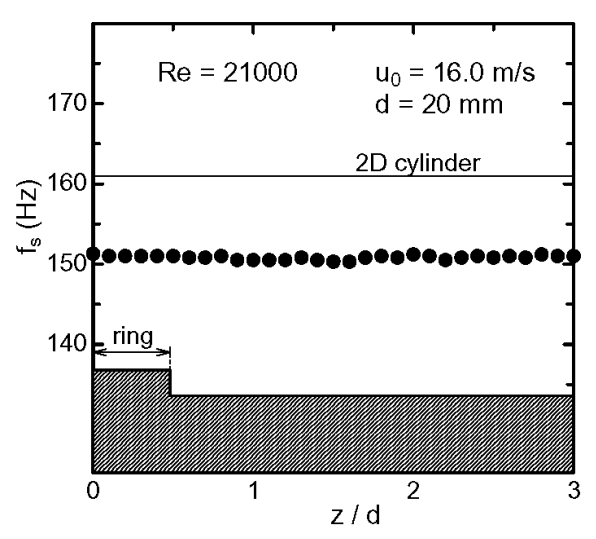

(b) $R e_{d}=21000$

Fig. 10 Vortex shedding frequency against the spanwise position of the ring-attached cylinder

vortex shedding frequency against the spanwise position of the ring-attached cylinder measured at $x=0$ and $y / d=0.75$ on the ring $(0 \leq z / d \leq 0.5)$, and at $x=0$ and $y / d=0.6$ on the base cylinder $(0.5 \leq z / d \leq 3)$. At $R e_{d} \approx 10000$, the vortex shedding frequency $f_{s}$ at the position far from the ring $(2 \leq z / d \leq 3)$ is $82 \mathrm{~Hz}$, which almost corresponds to that for the $2 \mathrm{D}$ cylinder $\left(f_{s 2 \mathrm{D}}=82.5 \mathrm{~Hz}\right)$. However, at around the ring $(0 \leq z / d<2)$, there are two distinct peaks at $f_{s 1}=82 \mathrm{~Hz}$ and $f_{s 2}=74 \mathrm{~Hz}$ due to the vortex shedding behind the base cylinder and the ring, respectively. The similar characteristics were reported for the cylinders jointed with a step in the diameter ${ }^{(15)}$, which has twin peaks in the power spectrum of the velocity fluctuation around the step. The Strouhal numbers for the base cylinder and the ring at $R e_{d} \approx$ 10000 are $S t_{d}=f_{s 1} d / u_{0}=0.205$ and $S t_{D}=f_{s 2} D / u_{0}=0.239$, respectively. The higher value of the Strouhal number for the ring can be explained by the facts that the separation point for the ring slightly moves backward compared to that for the base cylinder, as shown in Fig. 5 (a) and Fig. 7 (a), and thus, the wake width narrows to the same level as that for the base cylinder, as shown in Fig. 11 (b) and (c).

In contrast, there is only a single shedding frequency at $R e_{d} \approx 20000\left(f_{s}=151 \mathrm{~Hz}\right)$, regardless of the spanwise position. This may be due to the equalization of the shedding frequency between the base cylinder and the ring. Otherwise, it may be due to the disappearance of the vortex shedding behind the ring. It is not immediately clear why there is only a single shedding frequency. The similar trend was also observed for the wavy cylinder investigated by Lam et al. ${ }^{(10)}$. That is, the vortex shedding had a single frequency regardless of the spanwise position of the wavy cylinder, while its diameter changed in a sinusoidal manner along the span. If the reason for this is supposed to the equalization of the shedding frequency, the Strouhal number for the base cylinder $\left(S t_{d}=f_{s} d / u_{0}=0.189\right)$ is lower and that for the ring $\left(S t_{D}=f_{s} D / u_{0}=0.244\right)$ is higher than that for the 2D cylinder $\left(S t_{d}\right.$ $\left.=f_{s 2 \mathrm{D}} d / u_{0}=0.202\right)$. These are consistent with the fact that the separation point for the base cylinder moves forward and that for the ring moves backward, as shown in Fig. 5 (b) and Fig. 7 (b).

\section{Suppression of Vortex Shedding}

\subsection{Fluctuating velocity}

Figure 11 shows contours of the fluctuating velocity $u_{r m s} / u_{0}$ behind the cylinder in an $x-y$ plane measured using an I-type hot wire probe. The distribution of $u_{r m s} s u_{0}$ at the wake center $(y=0)$ is shown in Fig. 12. Although the value of $u_{r m s}$ measured using the hot wire probe is not correct due to the variation in the flow direction in the separated flow region, we can discuss the qualitative value as follows. The value of $u_{r m s} / u_{0}$ for the $2 \mathrm{D}$ cylinder in Fig. 11 (a) is high at $|y / d|=0.5-0.6$ due to the fluctuation of the shear layer that has 

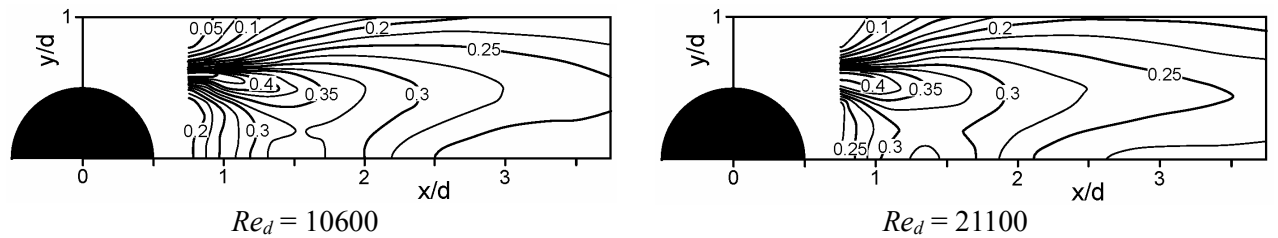

(a) $2 \mathrm{D}$ cylinder
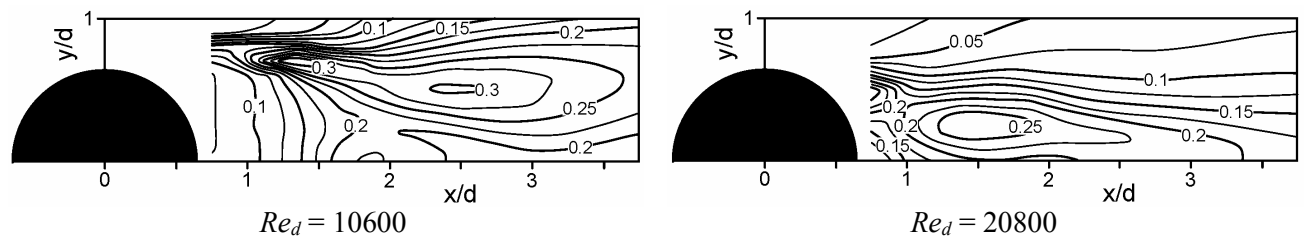

(b) ring attached cylinder at $z / d=0$ (behind the ring); $D / d=1.3, W / d=1.0, P / d=6$
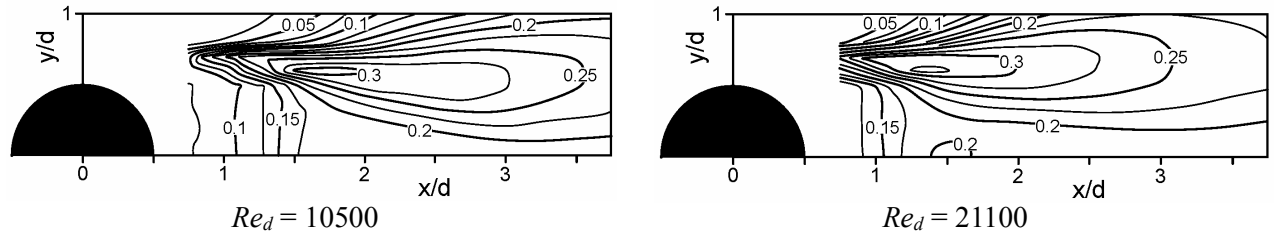

(c) ring attached cylinder at $z / d=3$ (behind the base cylinder); $D / d=1.3, W / d=1.0, P / d=6$

Fig. 11 Contours of the fluctuating velocity $u_{r m s} / u_{0}$ behind the cylinder for $L / d=18$

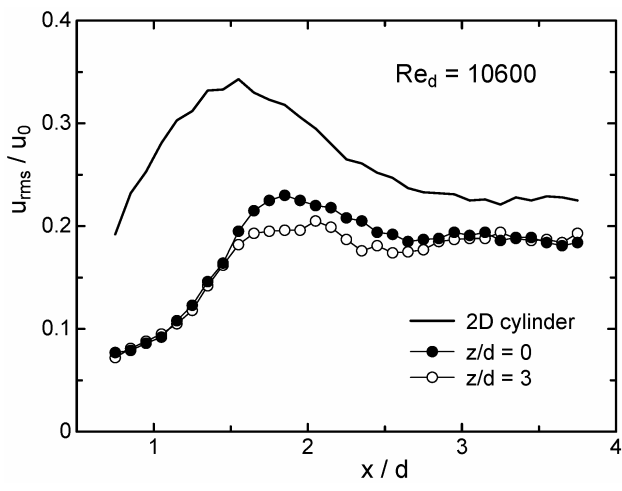

(a) $R e_{d}=10600$

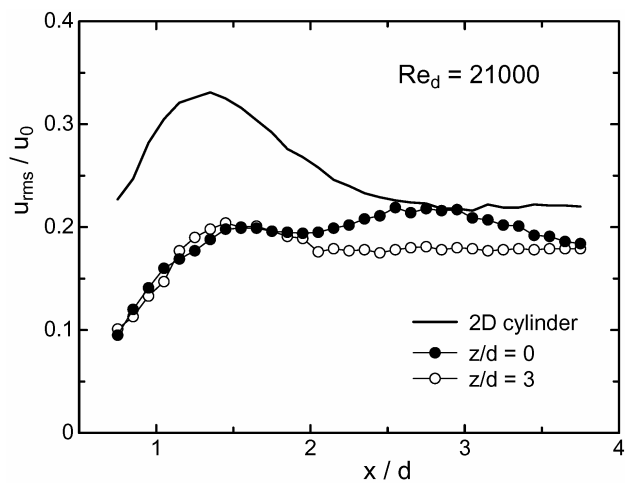

(b) $R e_{d}=21000$

Fig. 12 Fluctuating velocity distributions at the wake center $(y=0)$ for $L / d=18$

separated from the sides of the cylinder. The shear layer rolls up and across the wake center at $x / d=1.4-1.5$, which is where the value of $u_{r m s} / u_{0}$ reaches a maximum, as shown in Figs. 11 (a) and 12. In contrast, Figs. 11 (b) and (c) reveal that the value of $u_{r m s} / u_{0}$ decreases dramatically when the rings are attached for both $R e_{d} \approx 10000$ and 20000, particularly directly behind the cylinder $(x / d<1)$. This indicates that the shear layer fluctuation is weakened when the rings are attached, leading to the flattening of the maximum $u_{r m s} / u_{0}$ value, as shown in Fig. 12.

\subsection{Fluctuating lift}

As shown earlier in Fig. 7, a weakening of the fluctuating velocity accompanies the weakening of the surface pressure fluctuation when the rings are attached to the cylinder. Figure 13 displays time traces of the fluctuating surface pressures measured at $\phi= \pm 90^{\circ}$. For the 2D cylinder, the pressures fluctuate vigorously at the shedding frequency in a sinusoidal pattern. This causes a large fluctuating lift to act on the cylinder. In contrast, the fluctuations for the ring-attached cylinder $\left(z / d=0,1\right.$ and 3) are much weaker at both $R e_{d} \approx 10000$ and 20000 , indicating a significant suppression of the vortex shedding. 


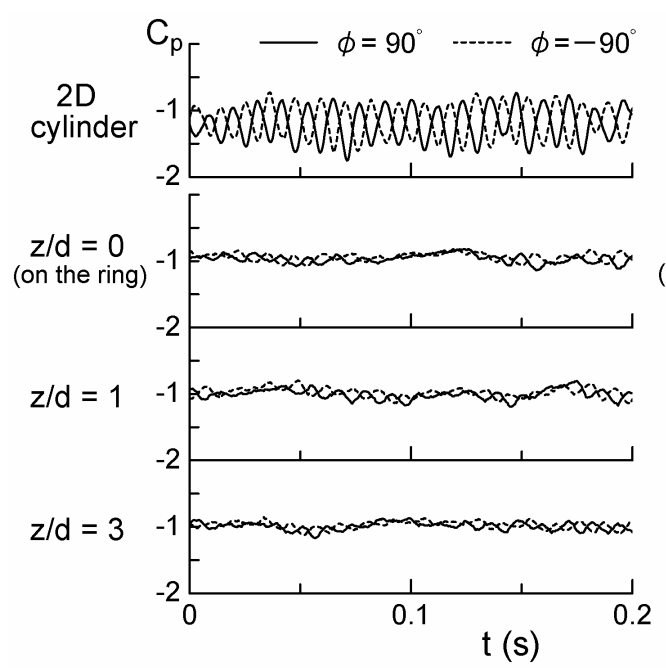

(a) $u_{0}=8.1 \mathrm{~m} / \mathrm{s}, R e_{d}=10200$

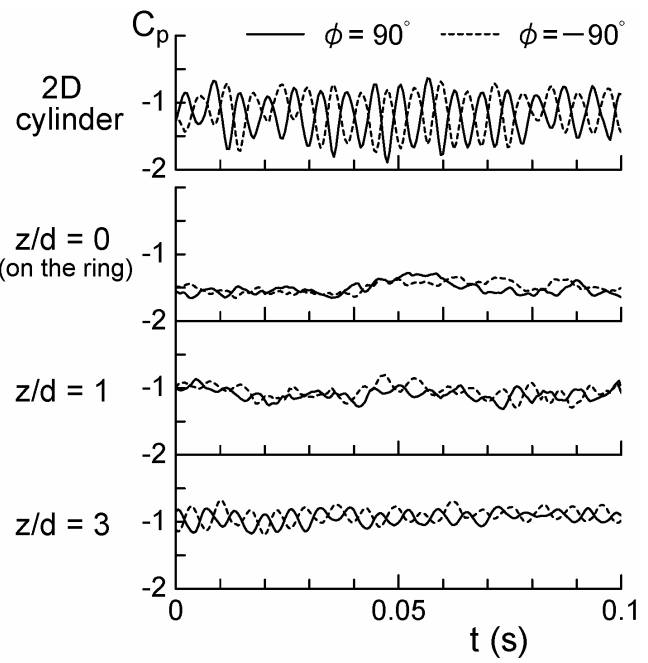

(b) $u_{0}=16.2 \mathrm{~m} / \mathrm{s}, R e_{d}=20400$

Fig. 13 Time traces of the fluctuating pressure coefficient at $\phi= \pm 90^{\circ}$ $(L / d=18, D / d=1.3, W / d=1.0, P / d=6)$

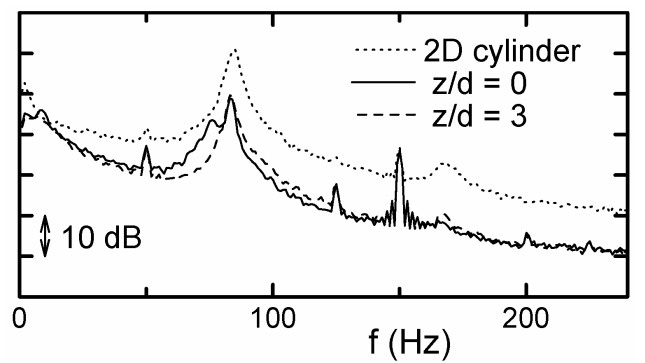

(a) $u_{0}=8.1 \mathrm{~m} / \mathrm{s}, R e_{d}=10200$

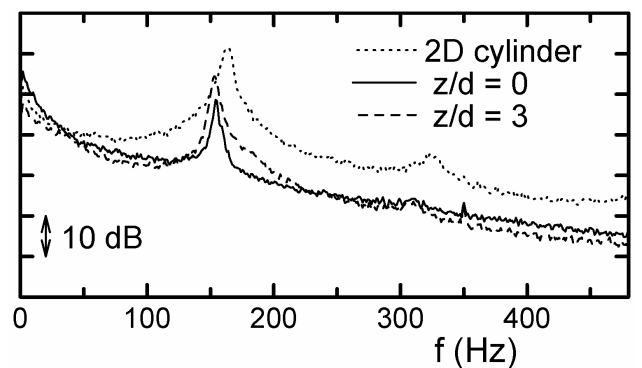

(b) $u_{0}=16.2 \mathrm{~m} / \mathrm{s}, R e_{d}=20400$

Fig. 14 Power spectrum of the fluctuating pressure coefficient at $\phi=90^{\circ}$ $(L / d=18, D / d=1.3, W / d=1.0, P / d=6)$

Figure 14 shows a power spectrum of the fluctuating surface pressure at $\phi=90^{\circ}$. There is a main peak at the shedding frequency $\left(f_{s}=74-82.5 \mathrm{~Hz}\right.$ at $R e_{d}=10200$ and $f_{s}=151-$ $161 \mathrm{~Hz}$ at $R e_{d}=20400$ ) and additional peaks at $f=50,125$, and $150 \mathrm{~Hz}$ that can be attributed to the $\mathrm{AC}$ power supply and vibrations from a blower. For both $R e_{d} \approx 10000$ and 20000 , the fluctuating pressure for the ring-attached cylinder is approximately $10 \mathrm{~dB}$ lower than that for the 2D cylinder, except in the low frequency range $\left(f<50 \mathrm{~Hz}\right.$ for $R e_{d}=10200$, and $f<100 \mathrm{~Hz}$ for $R e_{d}=20400$ ). The explanation for the fluctuating lift suppression is not readily apparent, but may be related to the formation of streamwise vortices at both ends of the ring, which is shown in Fig. 8. Owen et al. ${ }^{(4)}$ and Darekar and Sherwin ${ }^{(5)}$ also assumed that the streamwise vortices, which were generated by three-dimensional surface modifications, play a dominant role for the suppression of vortex shedding, although no evidence was depicted. Further study is necessary to clarify the reason behind the vortex shedding suppression.

\section{Conclusions}

Cylindrical rings were attached along the span of a cylinder at an interval of several diameters to reduce the drag and fluctuating forces caused by fluid flow. Experiments were performed at Reynolds numbers based on the cylinder diameter $d$ ranging between $R e_{d}=$ 3000 for 38000 . The aspect ratio of the cylinder, $L / d$, was approximately 20 . 
(1) The experimental results revealed that the drag force on the ring-attached cylinder was lower than for the 2D cylinder, even though the projected area was higher. The optimum ring configuration for drag reduction was found to be $D / d=1.3, W / d=1.0$, and $P / d=6$ at $R e_{d} \approx 30000$, where $D$ is the ring diameter, $W$ is the spanwise width of the ring, and $P$ is the spanwise pitch of the ring. This configuration reduced the drag force by $15 \%$.

(2) The considerable drag reduction was attributed to the formation of separation bubbles on both sides of the ring in the $R e_{d}>10000-20000$ range. This, in turn, led to the narrowing of the wake behind the ring and the pressure recovery at the rear of the ring.

(3) The fluctuating lift, which was estimated from the fluctuating surface pressures, was also reduced for all Reynolds numbers examined due to the suppression of vortex shedding.

\section{References}

(1) Williamson, C.H.K., Three-Dimensional Wake Transition, J. Fluid Mech. Vol.328 (1996), pp.345-407.

(2) Bays-Muchmore, B. and Ahmed, A., On Streamwise Vortices in Turbulent Wakes of Cylinders, Phys. Fluids A Vol.5, No.2 (1993), pp.387-392.

(3) Bearman, P.W. and Owen, J.C., Reduction of bluff-body drag and suppression of vortex shedding by the introduction of wavy separation lines, J. Fluids and Structures Vol.12 (1998), pp.123-130.

(4) Owen, J.C., Bearman, P.W. and Szewczyk, A.A., Passive control of VIV with drag reduction, J. Fluids and Structures Vol.15 (2001), pp.597-605.

(5) Darekar, R.M. and Sherwin, S.J., 2001. Flow Past a Square-Section Cylinder with a Wavy Stagnation Face, J. Fluid Mech. Vol.426 (2001), pp.263-295.

(6) Scruton, C., and Walshe, D.E.J, A Means for Avoiding Wind-Excited Oscillations of Structures with Circular or Nearly Circular Cross Section, Natl, Phys. Lab. (U.K.), Aero Rep., 335(1957).

(7) Nakagawa, K., Fujino, T., Arita and Y., Shima, T, An Experimental Study of Aerodynamic Devices for Reducing Wind-Induced Oscillatory Tendencies of Stacks, Proc. Symp. Wind Effects on Buildings and Structures, Teddington, (1963), pp. 774-795.

(8) Ahmed, A. and Bays-Muchmore, B., Transverse Flow Over a Wavy Cylinder, Phys. Fluids A Vol.4, No.9 (1992), pp.1959-1967.

(9) Ahmed, A., Khan, M.J. and Bays-Muchmore, B., Experimental Investigation of a Three-Dimensional Bluff-Body Wake, AIAA Journal Vol.31, No.3 (1993), pp.559-563.

(10) Lam, K., Wang, F.H., Li, J.Y. and So, R.M.C., Experimental Investigation of the Mean and Fluctuating Forces of Wavy (Varicose) Cylinders in a Cross-Flow, J. Fluids and Structures Vol.19 (2004), pp.321-334.

(11) Lam, K., Wang, F.H. and So, R.M.C., Three-Dimensional Nature of Vortices in the Near Wake of a Wavy Cylinder, J. Fluids and Structures Vol.19 (2004), pp.815-833.

(12) Norberg, C., An Experimental Investigation of the Flow around a Circular Cylinder: Influence of Aspect Ratio, J. Fluid Mech. Vol.258 (1994), pp.287-316.

(13) Nakamura, H. and Igarashi, T., Unsteady Heat Transfer from a Circular Cylinder for Reynolds Numbers from 3000 to 15000, Int. J. Heat and Fluid Flow Vol.25, No.5 (2004), pp.741-748.

(14) Yagita, M., Kojima and Y., Matsuzaki, K., A Study on the Wake of a Stepped Circular Cylinder (in Japanese), Transactions of the Japan Society of Mechanical Engineers, Series B, Vol.49, No.443 (1983), pp.1338-1344.

(15) Norberg, C., An Experimental Study of the Flow around Cylinders Jointed with a Step in the Diameter, 11th Australasian Fluid Mech. Conf., (1992), pp.507-510. 